\title{
Remarques sur les exploitations rurales en Touraine au haut Moyen Âge
}

Sho-ïchi Sato

\section{(2) OpenEdition}

\section{Journals}

Édition électronique

URL : http://journals.openedition.org/abpo/1209

DOI : $10.4000 /$ abpo.1209

ISBN : 978-2-7535-1495-9

ISSN : 2108-6443

Éditeur

Presses universitaires de Rennes

Édition imprimée

Date de publication : 20 septembre 2004

Pagination : 27-36

ISBN : 978-2-7535-0053-2

ISSN : 0399-0826

\section{Référence électronique}

Sho-ïchi Sato, «Remarques sur les exploitations rurales en Touraine au haut Moyen Âge », Annales de Bretagne et des Pays de l'Ouest [En ligne], 111-3| 2004, mis en ligne le 20 septembre 2006, consulté le 01 mai 2019. URL : http://journals.openedition.org/abpo/1209 ; DOI : 10.4000/abpo.1209 


\title{
Remarques sur les exploitations rurales en Touraine au haut Moyen Âge
}

\author{
Sho-ïchi SATO \\ Professeur d'histoire médiévale à l'Université de Nagoya \\ (Institute for the advanced research)
}

Comme le titre l'indique, nous allons discuter du régime agraire qui prévalait en Touraine durant le Haut Moyen Âge, tâche difficile en l'occurrence. D’une manière générale, tous les historiens de cette époque éprouvent un sentiment de frustration, né de la pauvreté de la documentation disponible, mais les historiens de la Touraine auraient des raisons particulières de se plaindre, car on sait que les archives de l'abbaye de Saint-Martin de Tours, qui formaient un des ensembles les plus riches et les plus prestigieux de l'Europe médiévale et avaient déjà souffert des déprédations des Normands vers les années 860 , ont été détruites de manière systématique à la Révolution ${ }^{1}$. Pour la période antérieure à l'an mil, ne subsistent que des copies de chartes tardives. Comment ne pas déplorer ces disparitions massives de documents?

Si on limite les investigations à la France d'aujourd'hui et aux contrées voisines (Ille-de-France, Picardie, Champagne, Ardenne, Provence), on dispose des polyptyques d'Irminon, de Saint-Bertin, de Saint-Remi, de Montiérender, de Prüm et de Saint-Victor de Marseille, pour ne mentionner que plus importants, qui constituent des sources d'information particulièrement riches ${ }^{2}$. Malheureusement, l'abbaye de Saint-Martin n'a pas eu la chance de conserver et de transmettre le polyptyque (discriptio) rédigé dans les derniers jours d'Alcuin et l'autre polyptyque (descriptio villarum

1. Cf. la contribution de M. Mersiowsky au présent volume : «Saint-Martin de Tours et les chancelleries carolingiennes ", infra p. 73-90.

2. Je me permets de citer ci-après leurs éditions plus ou moins récentes. Pour SaintGermain-des-Prés : D. Hagermann, K. Elmshauser et A. Hedwig, Das Polyptychon von SaintGermain-des-Prés, et l'étude qui la complète : K. Elmshauser et A. Hedwig, Studien zum Polyptychon von Saint-Germain-des-Prés. Pour Saint-Bertin : F.-L. GANSHOF, Le polyptyque de l'abbaye de Saint-Bertin... Pour Saint-Rémi de Reims, J.-P. Devroey, Le polyptyque et les listes de cens de l'abbaye de Saint-Réemi... Pour Montiérender, C.-D. DroEste, Das Polyptichon von Montiérender... Pour Prüm : I. SchWAB, Das Prümer Urbar. Enfin, pour SaintVictor de Marseille : B. Guerard, A. Marion et L. Delisle, Polyptyque de Saint-Victor de Marseille... 
sancti Martini) établi en 856 sous le règne de Charles le Chauve ${ }^{3}$. Si cette abbaye avait réussi à les préserver, ils auraient compté parmi les plus anciens de ces fameux polyptyques carolingiens mentionnés plus haut.

Mais, par rapport aux régions mentionnées ci-dessus, nous avons au moins l'avantage de pouvoir nous former quelques vues sur la vie agricole en Touraine au tout début du Moyen Âge, grâce à la découverte des documents comptables de Saint-Martin de Tours. Encore que fragmentaires, ces documents fournissent bien des informations utiles à notre propos.

\section{La question du régime domanial classique}

Tous les travaux sur le monde rural du Haut Moyen Âge reviennent sur ce problème : rencontre-t-on à cette époque le régime domanial classique? Ce prototype du domaine seigneurial s'organisait selon deux masses inégales : les tenures et la réserve seigneuriale (mansus indominicatus, etc.), dont l'exploitation reposait sur la main d'œuvre servile. Ces deux composantes se combinaient pour constituer cette forte structure que fut le domaine de type classique $^{4}$. Adriaan Verhulst a su merveilleusement débrouiller, il y a presque quarante ans, la complexité des conditions sociales et naturelles qui ont permis la genèse de ce type de domaine dans la partie septentrionale du bassin parisien au vII ${ }^{\mathrm{e}}$ siècle $^{5}$. Avec sagacité, il a établi que l'aire pédologique de sa propagation se limitait aux terres limoneuses à vocation céréalière ${ }^{6}$.

Comme ce type de seigneurie était censé former le système de production le plus efficace et le plus rentable, donc le plus avancé à l'époque, il est naturel que les historiens aient scruté de très près les sources locales afin de reconstituer la structure économique de la région concernée et de mesurer son développement à une échelle plus globale.

Si on revient à la Touraine, vu la rareté des documents conservés, on n'a guère de chance d'apercevoir un tel régime. Je voudrais cependant signaler deux travaux importants pour notre propos. Le premier est un article de Michel Rouche consacré à la géographie rurale au temps de Charles le Chauve $^{7}$. Il est vrai qu'il ne s'agissait pas d'une monographie sur la Touraine,

3. Pour la descriptio faite en 804, cf. L. LEVILlain (éd.), Recueil des actes de Pépin..., $\mathrm{n}^{\circ} 12$, p. 46 : Sed ipse Agenus advocatus et Magenarius prepositus ex ipso monasterio de presente adstabant et taliter incontra ipsos intendebant quod jam dictus abba nec ipsi nullas functiones nec redibutiones eis non exactaverant nec exactore jusserant, nisi quale ipsi per drictum vel per triginta annos partibus ipsius monasterii tam ipsi quam et eorum antecessores desolserant; et discriptionem ibidem optulerunt ad relegendum, in quo continebatur quomodo sub tempus Alcuino abbate ipsi coloni ex ipsa villa, ... ipsa discriptio, anno trigessimo quarto regnante Carolo rege. Pour celle de l'année 856 , la Chronique de Pierre, fils de Béchin écrit : Anno uerbi incarnati DCCCLVI Caroli autem XVII Hilduini abbatis III, facta est descriptio villarum sancti Martini (Chronicon Petri filii Bechini, p. 43).

4. On trouverait une des meilleures esquisses du régime domanial carolingien, toujours dans E. PERROY, Le monde carolingien, p. 21-51.

5. A. VERHULST, "La genèse du régime domanial classique... ", p. 150-152.

6. Ibid., p. 156-158.

7. Michel Rouche, " Géographie rurale du royaume de Charles le Chauve ", p. 193-205. 
mais l'auteur y affirme à plusieurs reprises que dans cette région, le régime domanial classique s'était déjà introduit sous Charles le Chauve au plus tard, la Touraine constituant la limite méridionale de son expansion ${ }^{8}$. La preuve est fournie, selon l'auteur, par une unité foncière locale nommée factus, la notion se rapportant en même temps aux exploitants qui en dépendaient. Il croit qu'elle équivaut au manse; et, comme le factus apparaît aux côtés d'une curtis dominica, nous tiendrions là l'indice d'une exploitation à structure bi-partie analogue au domaine classique. Cependant, l'auteur n'a pu avancer aucun argument permettant d'appréhender l'unité domaniale supérieure qu'auraient formée ces deux éléments. Car il est crucial d'établir un lien entre les manses et la curtis dominica. Ces deux éléments formaient-ils une structure domaniale organique, ou bien n'étaient-ils qu'une juxtaposition inorganique de terres de régimes différents? La première hypothèse serait établie, si l'on prouvait que les tenanciers des manses étaient astreints à des corvées au profit de la curtis dominica. Or, comme les actes royaux ne constituent pas une documentation de gestion, Michel Rouche n'a aucune chance d'y trouver la trace de la corvée, encore que cela ne signifie nullement qu'elle n'ait pas existé en Touraine ${ }^{9}$.

Il est vrai que, lorsqu'on rencontre dans une charte une expression paraissant désigner la corvée, il n'est pas facile de décider s'il s'agit d'une corvée à proprement parler ou bien d'un simple rappel des charges d'un tenancier. Il faut bien définir le cadre institutionnel et le contexte économique, dans lesquels s'insère une telle réquisition de la force de travail lorsqu'on essaye de définir la nature de la charge dans une charte. La question de la corvée étant ainsi envisagée, nous passons à l'analyse d'une charte de donation datée de 818, tirée de la Pancarte noire de Saint-Martin de Tours ${ }^{10}$.

Deux frères germains Haganon et Adiutor, tous les deux chanoines de Saint-Martin, ont promis de léguer à la communauté san-martinienne certains de leurs biens après leur décès à condition qu'à la mort de l'un des deux frères, le survivant en garderait l'usufruit. Il s'agit d'une donation post obitum, mais pas seulement, car les donateurs désignent, parmi les membres de la communauté canoniale, ceux qui auront le droit d'usage de

8. Ibid., p. 198.

9. Il serait opportun de nous rappeler ici que J. Nelson dans sa remarquable étude sur le règlement de conflits passé à la villa de Chasseneuil en Poitou en 828, n'a pas trouvé parmi les charges que s'assumaient les paysans dépendants de l'abbaye de Cormery sis à la villa d'Antoigné la moindre trace de la corvée, encore que cet auteur ait cru trouver dans la descriptio de Cormery dans laquelle les paysans dépendants ont été enregistrés, un instrument important pour la gestion du grand domaine classique auquel appartenait villa d'Antoigné. Voir J. NELSON, «Dispute settlement in Carolingian West Francia », p. 50, n. 18.

10. Bibliothèque nationale de France, ms. lat. Collection Baluze, tome $76, \mathrm{f}^{\circ} 328-329$ (MABILLE, La pancarte..., $\mathrm{n}^{\circ}$ 36, p. 82). Pour le texte imprimé, on peut consulter celui publié par Dom E. Martène et Dom D. Ursin dans leur Thesaurus novus anecdotorum, tome 1, Paris 1717, col. 20-23; il convient de compléter cette édition en ajoutant, après la datation d'après le règne de Louis le Pieux, la mention : Ego Geufredus clerici rogitus scripsi \& subscripsi. 
ces biens sous réserve qu'ils acquittent chaque année un loyer en argent à Saint-Martin. Vu les conditions imposées par les donateurs à l'abbaye, je tiendrais volontiers cette charte, non pas pour une donation mais plutôt pour une concession emphytéotique différée, et devant entrer en vigueur post obitum des signataires. Je signale, au passage, que ce type de contrat est attesté sous le nom de mainferme entre le début du $\mathrm{x}^{\mathrm{e}}$ siècle et le dernier quart du $\mathrm{XI}^{\mathrm{e}}$ siècle dans la Touraine, le Vendômois, la région de Chartres, la Beauce et le Poitou septentrional, dans les régions de défrichement ${ }^{11}$.

Revenons à la charte de l'an 818. On y trouve mentionnés, comme objets du legs, six ensembles fonciers répartis dans le Blésois, la Touraine et l'Anjou. Sauf une vigne sise dans la villa Sileariolas, aux environs de Montlouis près de Tours, les cinq autres exploitations étaient qualifiées, chose fort intéressante, de mansus dominicatus ou de mansus indominicatus. On a notamment un mansus dominicatus de la villa Pauliaco en Anjou, à la mise en culture duquel la quasi-totalité des tenanciers étaient astreints, à concurrence de la moitié de la vigne et des terres arables. Il est stipulé qu'on ne doit requérir d'eux aucune autre charge. Etait-ce une corvée imposée en contrepartie des tenures? On ne peut pas exclure cette possibilité, cependant, il me paraît qu'il ne s'agit que d'une lot-corvée, laquelle ne relève pas de la corvée proprement dite, mais plutôt de la mise en culture forcée d'un petit lopin de terre bien précisé ${ }^{12}$.

D'ailleurs, tout au long de cette charte, nous rencontrons des paysans relevant de deux statuts personnels distincts, dont l'un concerne des paysans quos colonario ordine vivere censuimus, pour citer la charte. Nous les retrouvons dans cinq des six unités d'exploitation rurale citées. Nous rencontrons aussi souvent des tenanciers qui a nobis ingenuitates promerverunt vel alibi delegati sunt ${ }^{13}$ " ceux qui ont acquis de nous la liberté ou ont été assignés à un autre lieu ". On n'y voit jamais des paysans de condition servile, puisqu'il n'y a aucune allusion au mancipium ${ }^{14}$. La charte de donation de 818 jalonne-t-elle une évolution particulière de la structure domaniale? Si elle atteste un régime domanial en voie de dislocation, le phénomène serait singulièrement précoce car l'Île-de-France, qui est le berceau du régime domanial classique, est encore en pleine prospérité.

11. P. Gasnault, "Les actes privés... ", p. 50; A. Chedeville, Chartres et ses campagnes..., p. 117-118; D. Barthelemy, La société dans le comté de Vendôme..., p. 44-47; S. Sato, "Chartes de mainferme et la communauté religieuse... ", p. 122-139.

12. Paris, Bibliothèque nationale de France, Collection Baluze, tome $76, \mathrm{f}^{\circ} 328 \mathrm{v}^{\circ}: E t$ constituimus illos homines in omnibus praedictis locis commanentes illam terram \& vineas \& omnia ad medietatem collaborare, \& nihil aliud eis requiratur nec post nos nihil inquietudines portiantur.

13. Ibid.

14. Il est vrai que l'auteur, comme E. Renard, a mis en doute la théorie établie sur la classification de différents statuts serviles. Cf. E. RENARD, " Lectures et relectures d'un polyptyque carolingien... ", p. 374-435; Id., "Les mancipia carolingiens étaient-ils des esclaves?..." ", p. 179-209. 
La question essentielle est donc bien de savoir si les mansi dominicati que Haganon et Adiutor avaient organisés dans le Val de Loire étaient intégrés à cette structure d'exploitation bi-partie que l'on appelle le régime domanial classique, ou si cette expression ne représente qu'une entité indépendante. Pour qu'il y ait régime domanial classique, il faut une réserve seigneuriale entretenue par une force de travail servile, sans quoi ce système ne fonctionne plus. Or, nous avons constaté tout à l'heure qu'on ne trouve pas l'ombre d'un esclave dans les mansi indominicati des chanoines san-martiniens. Il faut donc préciser en quoi consistaient ces mansi.

La même charte de donation offre des indices permettant de poursuivre la discussion. Comme je l'ai dit plus haut, elle portait sur cinq mansi dominicati, un dans le Blésois, deux en Touraine et les deux derniers en Anjou. Trois d'entre eux, ceux qui se trouvent dans les régions de Blois et Angers, étaient revenus à Haganon et à Adiutor à titre d'héritage (mansum nostrum dominicatum quam vestra consoltia fratrum in hereditate habemus... ou ... cedimus mansum dominicatum ex nostra hereditate portiones nostras ${ }^{15}$ ). Sur les deux qui sont en Touraine : l'un se trouve in pago Toronico, in condita Monte Laudeacinse, in villa Grussio, et l'autre in villa Braico. Il est intéressant de trouver, dans la description du mansus dominicatus placé dans la villa de Greux aux environs de Montlouis, la phrase suivante : mansus dominicatus quem de diversis hominibus pariter comparavimus... Il s'agit d'un regroupement de terrains que les deux donateurs avaient achetés à diverses personnes. Même cas pour l'autre, c'est-à-dire le mansus indominicatus in villa Braico. Ce dernier manse se composait de lopins qu'ils avaient acquis de diverses personnes : in ipso pago in villa Braico mansum indominicatum de diversis hominibus contractum. Le sens du mot contractum est, il est vrai, un peu vague, néanmoins il est permis de l'interpréter comme " acquis par achat ", d'après le parallélisme de la formulation adoptée pour la villa de Greux.

L'image du mansus indominicatus qui ressort de la charte de donation de Haganon et Adiutor me paraît difficilement conciliable avec celle de la réserve seigneuriale d'un seul tenant de la théorie domanialiste. Au lieu de suggérer une entité organique homogène et compacte entretenue par une force de travail servile, elle décrit plutôt un cadre terrien formé par l'agrégation de lopins de terre d'origine et de statut juridiques divers. Cette présomption se renforce quand on rencontre, toujours dans la même charte, à la villa Cresciaco en Anjou, un mansus dominicatus dans lequel un certain Theodovinus possédait un petit manse (mansellum) ${ }^{16}$. Une inclusion analogue se retrouve in villa Pauliaco en Anjou ${ }^{17}$.

15. Paris, Bibliothèque nationale de France, Collection Baluze, tome $76, \mathrm{f}^{\circ} 328$.

16. Ibid., $\mathrm{f}^{\circ} 328 \mathrm{v}^{\circ}$ : ... in villa Cresciaco, excepto illud quod de Gunduino comparavimus, et illum mansellum quem Teodovinus tenevit.

17. Ibid. : ... in villa Pauliaco, ... excepto... et illam terium partem in illo manso ubi Sicfredus commanentibus, illum campum qui dicitur ad Gumonas et ad illa pratella. 
Ainsi, d'après le contexte de la charte de donation de Haganon et Adjutor en 818 , nous devons voir dans le mansus indominicatus du Val de Loire une entité tout à fait différente d'une réserve seigneuriale. Que signifiaient donc les mots mansus indominicatus, et encore villa, dans cette région? Il faut rappeler ici la constatation qu'Élisabeth Lorans a faite naguère à propos de la villa carolingienne dans la région de Loches, en Touraine méridionale. C'est la deuxième contribution importante que nous voudrions signaler pour l'étude rurale de la Touraine. Elle écrit : " La villa (c'est-à-dire dans cette région) ne répond pas au schéma classique du "grand domaine". Il s'agit avant tout d'un espace de référence qui peut inclure des manses, des parcelles mais aussi des alleux, qui tous jouissent d'une grande autonomie par rapport à la villa ${ }^{18}$. "On doit ajouter une remarque allant dans le même sens formulée récemment par Olivier Bruant en conclusion de son étude de perspective plus large sur la villa carolingienne ${ }^{19}$. Il caractérise la villa de Courçay en Lochois, plutôt qu'un grand domaine, comme une seigneurie en voie de développement et de mutation, sur laquelle un seigneur proto-banal pouvait étendre son autorité ${ }^{20}$. Aucun des deux, alors qu'ils se sont interrogés tous les deux sur la villa carolingienne en Touraine, n'admet qu'il s'agit d'un "grand domaine " au sens exact du mot, ni à plus forte raison d'un grand domaine à structure bi-partie.

\section{Prédominance de l'exploitation colongère}

En effet, dans la structure agraire qui prévalait en Touraine pendant le Haut Moyen Âge, le grand domaine à proprement parler était absent ou marginal. Pour restituer un état plus ancien de la structure agraire dans cette région, les documents comptables de Saint-Martin de Tours, datés de la fin du VII ${ }^{\mathrm{e}}$ siècle, constituent un corpus de données inestimable, que j'ai étudié pendant plusieurs années et sur lequel j'ai publié, il y a presque six ans, une thèse écrite en japonais ${ }^{21}$.

Ces données comptables, enregistrées sur les vingt-huit fragments de parchemin, livrent cent vingt-six toponymes dont seize sont dépourvus de détermination du fait de mutilations affectant le début des lignes. Sur les cent dix échantillons restants, les quatre-vingt-dix-sept comportent la détermination colonica ou ses abréviations col ou colonec. Le reste est soit villa soit domus. Si on se fonde sur ces données, qui décrivent d'ailleurs une aire géographique s'étendant à peu près à toute la Touraine, on peut soutenir que cette région formait à la fin du VII ${ }^{\mathrm{e}}$ siècle, un bassin d'exploitation colongère ${ }^{22}$.

18. E. LORANS, Le Lochois..., p. 48.

19. O. BRUAND, " La villa carolingienne : une seigneurie?... ", p. 349-373.

20. Ibid., p. 369.

21. S. SATO, Une abbaye et ses paysans...; sur les documents comptables de Saint-Martin de Tours, cf. également Idem, "The Merovingian accounting documents..." .

22. F.-L. GANSHOF, "Quelques aspects principaux de la vie économique... ", p. 82, invite à considérer la colonica dans une perspective de son développement vers le manse sans qu'il ait rendu compte son origine ni sa physionomie, tandis que J.P. PolY et É. BouRnAZEL, La mutation féodale... p. 357, cherchent ses origines dans de grands fundi du Bas-Empire romain. 
Étant donné que l'économie agricole de l'abbaye de Saint-Martin reflète le régime d'exploitation général en Touraine, il est légitime de poursuivre notre réflexion sur le régime agraire de la Touraine en continuant à faire fond sur le même dossier.

Dans le système san-martinien, une colonica était un agrégat de ménages paysans de nombre variable (d'un à quarante-neuf, mais la plupart des cas se situent entre trois et douze ménages). Ce sont les circonscriptions de base du domaine d'ensemble de Saint-Martin de Tours. Il me semble que la colonica n'a pas de fondement fiscal et qu'elle n'a rien à voir avec une seigneurie. Entre les quatre-vingt-dix-sept colonicae, on ne distingue pas de hiérarchie fonctionnelle. Il n'y avait que des instances intermédiaires, c'est-à-dire les domus, qui reliaient des colonicae et le bureau des comptes de l'abbaye pour la collecte des prélèvements. Les exploitations paysannes ne révèlent aucune organisation domaniale sous quelque forme que ce soit. Ce système n'est pas autre chose qu'une juxtaposition d'exploitations familiales. Je crois pouvoir soutenir, sans grand risque d'erreur, que ce type d'organisation était la structure agraire dominante dans les campagnes tourangelles à la fin du VII ${ }^{e}$ siècle. Sur de telles bases, s'amorçait cependant, en des endroits ménageant des conditions naturelles plus favorables ou par le jeu d'influences extérieures, un développement d'exploitations plus complexes et peut-être de plus grande dimension ${ }^{23}$.

On observe déjà, dans les documents comptables, des traces précoces de cette évolution. Il s'agit des villae des fragments $n^{\circ} 18$ et surtout $n^{\circ} 19$. Dans le dossier de Saint-Martin, curieusement, on rencontre en effet cinq fois des lieux-dits appelés, non pas colonica, mais villa. Cette terminologie concerne surtout le $\mathrm{n}^{\circ} 19$, qui, à ce titre, pourrait se rapporter à une localité où l'on trouve des exploitations en pleine mutation sociale grâce aux défrichements et à l'ouverture de nouveaux espaces agricoles, avec une tendance à la constitution de grands domaines de structure complexe. Comme le regretté Adriaan Verhulst l'a parfaitement démontré, la mise en exploitation de terres en friche fut l'un des éléments essentiels de la création du nouveau système domanial ${ }^{24}$. On relèvera à cet égard que les villae du document $\mathrm{n}^{\circ} 19$ appartenaient à la catégorie des domaines de SaintMartin de Tours qui s'acquittaient le plus souvent de redevances en bois (lignaticum < lignum). On aurait toléré que les paysans de ces villae s'acquittent de leurs charges au moyen du bois dont ils disposaient facilement grâce à leurs activités de défricheurs au lieu des céréales qu'ils ne produisaient pas encore ${ }^{25}$. D'après les tentatives de localisation, les toponymes du fragment $\mathrm{n}^{\circ} 19$ se trouveraient dans une région assez proche de Blois,

23. O. BRUAND, " La villa carolingienne : une seigneurie?... ", p. 361, n. 36.

24. A. VERHULST, "La genèse du régime domanial classique...", p. 149 sqq.

25. À ce point-ci, Jean Durliat pense que la prestation en bois était acquitté effectivement en grain par les contribuables. Voir J. DuRLIAT, "Qu'est-ce qu'un polyptyque?... ", p. 131 . 
en marge de la plaine de Beauce qui était pour l'historien gantois à la périphérie de la région de propagation du régime domanial classique ${ }^{26}$.

Je n'ai pas l'intention de m'étendre sur le grand débat relatif au défrichement et au peuplement de la plaine de Beauce, qui impliqua entre autres Ferdinand Lot, Camille Jullian et Marc Bloch. Je reprendrai simplement la conclusion de Adriaan Verhulst selon qui, en Beauce intérieure, le défrichement a commencé à l'époque franque. À la lumière de cette conclusion, les particularités du compte $\mathrm{n}^{\circ} 19$ témoignent d'une mutation précoce des structures agraires. Mais une fois admise une telle évolution pour la plaine de Touraine, il n'en subsiste pas moins des sujets d'interrogation : cette transition de l'exploitation colongère vers le domaine complexe a-t-elle atteint son terme? Quelle fut son aire d'extension? La structure agraire tourangelle a-t-elle conservé, malgré ce mouvement, quelque spécificité?

À ces questions, je répondrai provisoirement que, du fait de la pénurie de la force de travail servile disponible localement (condition indispensable à la constitution d'une réserve seigneuriale dans le système domanial classique), la réserve restait sans doute plutôt exceptionnelle. Dans l'aire de diffusion du grand domaine, soit au nord de la Seine, durant tout le VII ${ }^{\mathrm{e}}$ siècle, les esclaves affluaient sans discontinuer, qu'il s'agisse de prisonniers de guerre amenés par des seigneurs francs, ou de captifs rachetés par des religieux comme les saints Eloi, Philibert, Amand et Riquier ${ }^{27}$. Avec le développement des défrichements, la main d'œuvre servile abondante était la condition sine qua non pour la mise en place du domaine bi-parti, système où la réserve seigneuriale était l'élément peut-être le plus difficile à mettre en place, car il fallait la créer ex nihilo, au moyen d'esclaves d'introduction récente. Quant aux autres points, j'allèguerai, encore une fois, dans ma réponse, l'exemple d'Haganon et Adjutor. Sur leurs terres, le mansus indominicatus qui tenait lieu de réserve seigneuriale, constituait en réalité, comme nous l'avons vu, une juxtaposition de lopins de terre appartenant à des tenanciers différents et d'origines diverses. Il me semble qu'en Touraine, les éléments nécessaires pour les intégrer à une vraie formation domaniale ont manqué, surtout en raison de la faiblesse de la population servile.

\section{En guise de conclusion : \\ le domaine de Saint-Martin au temps d'Alcuin}

Pour clore cette brève communication, je voudrais ajouter quelques mots sur la fameuse lettre d'Élipand, archevêque de Tolède, adressée en 799 à Alcuin. Élipand l'écrivit dans un contexte de controverse religieuse, celui de l'hérésie adoptianiste, qui l'opposa à Alcuin ${ }^{28}$. Il s'adresse à lui sur

26. A. Verhulst, « La genèse du régime domanial classique... ", p. 149-151.

27. Vita Eligii, c. 10, p. 677; Vita Filiberti, c. 19, p. 12; Vita Amandi, I, c. 9, p. 435; Vita Richarii, c. 7, p. 448.

28. Sur la question théologique du conflit, voir W. HeIl, "Der Adoptionismus, Alkuin und Spanien ", p. 95-155. Pour la synthèse la plus récente il faudrait signaler J. C. CAVADINI, 
un ton particulièrement offensant, évoquant la richesse dont Alcuin aurait disposé comme chef de l'illustre abbaye. Il dit : " Prends garde, toi qui es notoirement connu pour posséder vingt mille esclaves, à ne pas concevoir d'orgueil, de ce fait, de ces richesses ${ }^{29}$. "C'est une phrase, comme on sait, qui a fait couler beaucoup d'encre, notamment en ce qui concerne l'interprétation des mots viginti milia servorum. Si Élipand dit vrai, les vingt mille esclaves correspondaient-ils à la totalité des gens de condition servile possédés par les monastères dont Alcuin cumulait l'abbatiat, c'est-à-dire, Ferrières, Saint-Josse-sur-Mer, Saint-Loup de Troyes et Saint-Martin de Tours? S'agissait-il uniquement des chefs de famille dépendant de ces abbayes ou bien ce chiffre incluait-il aussi les membres de leurs familles? Et si nous n'avons pas affaire à une pure invention de l'archevêque, comment s'est-il procuré ce chiffre? Je n'ai pas l'intention d'aller plus loin, signalant simplement que dans la deuxième moitié du viII ${ }^{\mathrm{e}}$ siècle, quelques influences wisigothiques s'exerçaient sur l'abbaye de Saint-Martin de Tours car cet établissement accueillait des clercs et des religieux espagnols qui avaient fui la domination arabo-musulmane ${ }^{30}$. On peut supposer que l'archevêque de Tolède tirait des informations de ce milieu, au premier chef sur la situation de l'abbaye tourangelle.

Il est fort probable que les vingt mille personnes dépendantes mentionnées par Élipand dans sa lettre à Alcuin descendaient des paysans enregistrés dans les documents comptables à la fin du VII ${ }^{\mathrm{e}}$ siècle. Ils n'étaient sans doute pas privés de liberté, mais, comme notre enquête le suggère, formaient une population rurale qui, sans dépendre personnellement de la seigneurie san-martinienne, devait payer une part des récoltes au titre de tenures colongères dont l'abbaye leur avait concédé l'exploitation, en se réservant un loyer. Les vingt mille paysans n'étaient probablement pas les cultivateurs forcés d'une réserve domaniale.

The Last Christology of the West... et également, D. A. Bullough, Alcuin. Achievement and Reputation, p. 419-429.

29. Alc. Ep. 182, p. 302 : Vide, ne tu sis ex illis, qui viginti milia servorum habere dinosceris, et ideo diviciis inflatus... À ce propos, cf. la contribution de M. Hartmann au présent volume : "Alcuin et la gestion matérielle de Saint-Martin de Tours ", infra p. 91-102.

30. En 799 dans une lettre destinée à Arn, archevêque de Salzbourg, Alcuin raconte qu'il a organisé une vie religieuse à Cormery où son prédécesseur Ithier en avait lancé son projet, en réunissant les moines qui y sont arrivés de Gothie comme réfugiés. Il est vrai qu'il s'agissait de l'abbaye de Cormery et non pas de Saint-Martin de Tours, mais la faible distance séparant les deux maisons religieuses et l'étroitesse des relations multiples qui reliaient une abbaye avec l'autre, me semblent rendre légitime notre présomption; cf. Alc. Ep. 184, p. 309. 


\section{RESUME}

La Touraine reste toujours un champ de recherche largement inculte, sinon vierge, pour l'histoire agraire du haut moye âge comme tant d'autres régions qui souffrent également de la rareté du document disponible. Nous avons lancé notre propre recherche en appliquant une question-critère bien connue : propagation ou absence du régime domanial classique dans ces terres situées à la croisée de plusieurs civilisations agraires? Notre enquête montre qu'il ne suffit pas de trouver les termes comme mansus indominicatus, curtis dominica etc. dans les documents pour constater une vraie introduction de ce régime, mais qu'il faut analyser de plus près en quoi consistaient de tels mansus indominicatus et curtis dominica. Un testament daté de 818 nous a permis de lancer l'hypothèse selon laquelle la Touraine n'a pas vu l'arrivée de ce régime en tant que tel et qu'elle resta largement un pays d'exploitation colongère.

\section{ABSTRACT}

The Touraine has been a research field that calls for much progress to be done, for rural and agricultural history of the early middle ages as other regions which suffered from the scarcity of documents. We launched into our own investigation for discovering the main features in land ownings and exploitations with a criterion : if the so-called bipartite classical manor did succeed to establish itself as rural organization on the soil of the Touraine, or not? Our investigations revealed that it was not enough to find out such terms as mansus indominicatus, curtis dominica for exemple, in the documents for asserting an installation of classical manor there, but to come to light with thorough investigations the entities which were qualified by the words mansus indominicatus and curtis dominica. A written will made in 818 permits us to make an assumption that the Touraine remained to be a virgin soil for the classical manor system and it prevailed there rather the colonical land ownings and exploitation during the early middle ages. 